\title{
ESTADO DE CONSERVACIÓN Y CONTEXTUALIZACIÓN CRONOLÓGICA DE LA COlección OsteológicA "GustaVo Le PAige"
}

\author{
Mark Hubbe, Macarena Oviedo y Christina Torres-Rouff ${ }^{1}$
}

"Insistimos que un estudio verdadero no termina una vez escrito".

Gustavo Le Paige (1974: 71).

\section{* Introducción}

Resumen

Entre 1950 y 1970 el sacerdote Gustavo Le Paige excavó una de las mayores colecciones osteológicas de América Latina, que ha llamado la atención de muchos investigadores en los últimos 40 años. En sus notas de terreno Le Paige consigna la exhumación de 5482 cráneos y cuerpos humanos, depositados en el Instituto de Investigaciones Arqueológicas y Museo de la Universidad Católica del Norte, en San Pedro de Atacama. Sin embargo, procesos acumulativos de abandono y falta de conservación preventiva produjeron serios daños en el estado de una parte significativa de esta colección, así como la pérdida de sus contextos. Aquí, presentamos el actual estado de conservación de la colección, al mismo tiempo que ofrecemos 50 nuevas dataciones radiocarbónicas que sitúan cronológicamente en forma más precisa a 21 de los cementerios más representativos de donde proviene nuestro objeto de estudio.

Palabras claves: dataciones radiocarbónicas - cementerios - restos humanos - períodos alfareros - cuenca de Atacama.

\section{Abstract}

Between the 1950 s and 1970 F Father Gustavo Le Paige excavated one of the largest osteological collections of Latin America, attracting the attention of numerous researchers over the past 40 years. In his field

notes, Le Paige recorded the excavation of 5482 skulls and human bodies deposited in the Instituto de Investigaciones Arqueológicas y Museo of the Universidad Católica del Norte (San Pedro de Atacama).

However, cumulative processes of abandonment and the lack of preventive conservation resulted in the loss of context and a decrease in the quality of preservation of a significant portion of this skeletal collection. Here, we present the current state of preservation of this collection, and 50 new radiocarbon dates that helps the chronological contextualization of 21 of the larger and more representative cemeteries in the Le Paige collection.

Key words: radiocarbon dates - human skeletal remains - cemeteries ceramic periods - Atacama.
En el primer número de esta revista, publicado en 1973, el padre Gustavo Le Paige realizó una síntesis de la colección que excavó entre 1955 y fines de la década de 1970, afirmando que era necesario "presentar a los lectores de la nueva revista un resumen de lo ya realizado para unirlo a las perspectivas y proyectos para el futuro en conexión con lo que se efectúa en el momento actual" (Le Paige 1973: 9). En la actualidad esta colección arqueológica es una de las más numerosas y valiosas de América Latina. Su importancia es especialmente acentuada al considerar el material osteológico que la conforma, el cual supera los cinco mil cráneos y cuerpos humanos que fueron excavados en casi un centenar de sitios arqueológicos en la región atacameña (cuenca del Salar de Atacama y río Loa). La colección osteológica fue de especial interés para Le Paige, quien publicó varios artículos y monografías sobre este material (p.e., Larraín et al. 1961, Le Paige 1964, 1966, 1977). En los 30 años que siguieron a su muerte ocurrida en 1980, esta colección ha sido resguardada en el Instituto de Investigaciones Arqueológicas y Museo (en adelante IIAM) de la Universidad Católica del Norte, la que ha sido estudiada por numerosos investigadores nacionales e internacionales. Sin embargo, la colección ha sufrido un proceso generalizado de abandono y pérdida de parte de sus contextos originales, perjudicando la inclusión de una parte significativa de ella en las investigaciones tanto bioantropológicas como arqueológicas. Por esta razón, nos parece importante presentar su actual estado de conservación así como la (re)contextualización del componente osteológico, para que de esta

Recibido: febrero 2011. Aceptado: mayo 2011.

${ }^{1}$ Instituto de Investigaciones Arqueológicas y Museo (IIAM) de la Universidad Católica del Norte, San Pedro de Atacama, CP 1410000, CHILE.Email:mhubbe@ucn.cl; Email: moviedo@ucn.cl; Email: ctorres@ucn.cl 


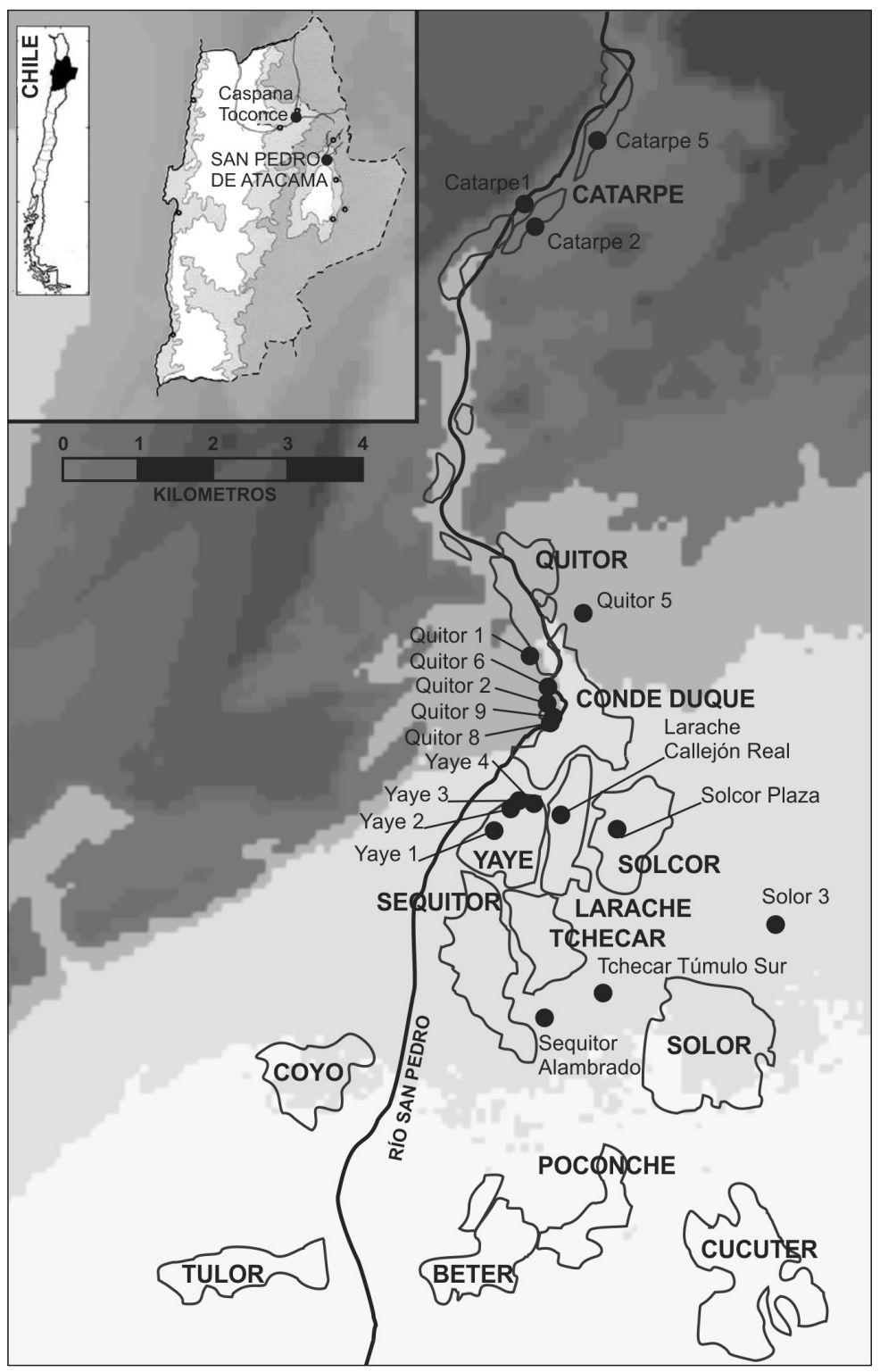

Figura 1. Mapa de la región atacameña indicando los ayllu y cementerios mencionados en el texto.

manera pueda servir de referencia a futuros estudios. Teniendo en mente este objetivo, en las próximas páginas presentaremos: a) una breve síntesis de las publicaciones que se han realizado sobre esta colección; b) su estado actual de preservación, y c) 50 nuevas dataciones radiocarbónicas directas que nos ayudarán tanto a contextualizar estos restos humanos como a situar cronológicamente - de manera precisa- los cementerios más representativos de donde provienen (Figura 1).
* Reseña de las investigaciones científicas REALIZADAS SOBRE LAS COLECCIONES OSTEOLÓGICAS DEL IIAM

Las publicaciones que abordan las colecciones osteológicas del IIAM se inician con Le Paige, quien además de obtenerlas, fue el fundador del Museo de San Pedro de Atacama (actualmente IIAM). Junto con distintos colaboradores, publicó una serie de monografías y estudios 
descriptivos sobre el material (Larraín et al. 1961; Le Paige y Larraín 1961; Le Paige 1964, 1966, 1968-69, 1972-73, 1973, 1974, 1977, 1978), siendo de especial importancia aquellos que pueden leerse en los Anales de la Universidad del Norte (1964) y en Estudios Atacameños (1973 y 1977). En ellos, presenta de manera sintética descripciones del contexto de los cementerios y de las tumbas, así como del número de tumbas en cada sitio y de los atributos craneométricos. Estos trabajos deben ser considerados el punto de partida de la puesta en valor científica de las colecciones osteológicas y, más aún, de la prehistoria atacameña. Le Paige empezó sus estudios científicos en abril de 1955 motivado por la gran cantidad de hallazgos funerarios y arqueológicos que realizó (Núñez 1993). En efecto, a mitad de ese año, inició salidas a terreno en las cuales excavó y documentó los contextos arqueológicos prácticamente en toda la región atacameña. Le Paige consideraba los cráneos como una fuente de información sin paralelo para entender la prehistoria atacameña $y$, es a causa de esto, que puso gran énfasis en la excavación y recuperación de los restos humanos, aunque a menudo sin los huesos post craneales. En este sentido, Le Paige fue más bien un perpetuador de prácticas de la antropología física del siglo XIX, la cual defendía la opinión de que el cráneo era la parte más informativa (si no la única) del esqueleto humano. En este punto, es necesario mencionar que las colecciones osteológicas de importantes museos del planeta formadas en este período están compuestas en gran medida por cráneos humanos sin post cráneo. Durante la década de 1960, Le Paige publicó tres trabajos de craneometría en los Anales de la Universidad del Norte (Larraín et al. 1961; Le Paige y Larraín 1961; Le Paige 1966), además de la síntesis en Estudios Atacameños (Le Paige 1973). La finalidad de estas publicaciones era la clasificación tipológica de la forma del cráneo en las antiguas poblaciones de los oasis atacameños, aunque se observa poco esfuerzo en interpretar las frecuencias de cada tipo craneano y su contribución hacia la comprensión del origen biológico de los grupos locales. Además de estos estudios, Le Paige también se refiere a los cementerios en otros trabajos $(1964,1977)$, contextualizando los restos humanos dentro del marco de la arqueología mortuoria.

En los años inmediatamente posteriores a la muerte de Le Paige, se produce una significativa descontextualización de la colección que había logrado formar, pues los restos humanos fueron separados de sus ajuares funerarios. Poco después, se forma el Instituto de Investigaciones Arqueológicas y Museo (IIAM), contratándose por primera vez arqueólogos investigadores que serían los responsables del patrimonio formado por Le Paige. En este período, existen algunos trabajos que intentan recuperar el contexto perdido de la colección, como por ejemplo, el de Jacobs (1986), quien observa que 58 de los 219 cuerpos momificados $(26,5 \%)$ depositados en el museo carecían de su contexto original, impidiendo a la autora establecer su asociación con las notas de Le Paige. Paralelamente a ese proceso, se incorporan al IIAM arqueólogos con formación académica, quienes promueven estudios de esos sitios arqueológicos, siguiendo métodos científicos actualizados, complementando de esta manera la colección osteológica con materiales provenientes de esos sitios (Costa 1985, 1988; Bravo y Llagostera 1986; Costa y Llagostera 1994). Estas nuevas excavaciones son instigadas por la propia descontextualización de la colección Le Paige y el hecho de que ésta se restringe mayoritariamente a los cráneos, lo que limita su utilidad en investigaciones con enfoques teóricos y metodológicos modernos. Las nuevas excavaciones fueron lideradas por María Antonietta Costa, Agustín Llagostera, Francisco Téllez y Leandro Bravo, y se enfocaron en la recuperación de los esqueletos y contextos completos, obteniéndose más de 200 individuos y sus ajuares mortuorios.

De especial interés para la colección osteológica fue la contratación de la antropóloga física María Antonietta Costa por parte del IIAM. Operando dentro de un marco basado en la osteobiografía (Saul 1972), Costa atrajo la atención de distintos grupos de investigación hacia las colecciones locales. Sus primeros trabajos, en la década de 1980, se enfocaron en cuestiones de estilo de vida y de la adaptación de la población prehistórica de los oasis a la vida cotidiana. Además, puso un énfasis en las relaciones de los atacameños con poderes foráneos, especialmente en el impacto que la influencia del Estado altiplánico de Tiwanaku tuvo sobre el estilo y la calidad de vida biológica de la población local (Costa 1985, 1988; Costa y Llagostera 1994). Aunque en este período los trabajos operaban dentro de un modelo más descriptivo, es posible ver las primeras investigaciones enfocadas en problemáticas relacionadas con la antropología física y la arqueología. Efectivamente, los trabajos iniciales de Costa sirvieron 
para destacar la importancia de la colección, llamando la atención de científicos nacionales e internacionales.

De ahí surgen, en la década de 1990, los primeros proyectos con apoyo de fondos nacionales e internacionales de largo plazo con consideraciones científicas más amplias en las colecciones osteológicas del IIAM. Junto con esto, se inicia la internacionalización de los estudios sobre las colecciones osteológicas. Estos se desarrollan dentro de algunas líneas específicas de trabajo de larga duración: craneometría, deformación craneana y estilo de vida. La primera línea de estudios liderada por José Cocilovo, Francisco Rotthammer y Héctor Varela sigue los trabajos de craneometría de Le Paige, pero ahora en un marco comparativo, aplicando técnicas estadísticas modernas. Esta línea de trabajo estuvo dirigida a reconstruir la historia biológica de las poblaciones locales y regionales (Rotthammer et al. 1986; Rotthammer y Silva 1990; Varela et al. 1990, 1993, 1995, 2008; Cocilovo et al. 1994; Costa et al. 1995; Varela y Cocilovo 1996; 2000, 2009; Varela 1997).

La segunda línea de trabajos también sigue un tema de interés para los primeros arqueólogos del país: la deformación craneana artificial (Henckel 1964; Le Paige 1964, 1973, 1977; Munizaga 1969). Como en la línea anterior, desde la década de 1990 se observa la apropiación de nuevas técnicas metodológicas y nuevos acercamientos teóricos que permiten la inclusión de los cráneos deformados de la colección en un contexto explicativo más amplio y relevante para el desarrollo de los conocimientos sobre el proceso de deformación craneana. En esta línea, los trabajos se dividen en dos categorías generales: aquellos que abordan los procesos que dieron origen a las deformaciones y sus consecuencias en el estudio de la variabilidad biológica de las poblaciones locales (Cocilovo y Zavattieri 1994; Cocilovo et al. 1995); y aquellos que abordan aspectos sociales y de construcción de identidad a través del estudio de la incidencia de los tipos de deformaciones artificiales (Torres-Rouff 2002, 2003, 2007, 2008, 2009; Torres-Rouff y Yablonsky 2005; Costa et al. 2008).

Además de estas dos líneas que siguieron intereses preexistentes, se desarrolló otra sobre estilo y calidad de vida local, con énfasis en el estudio de marcadores osteológicos de cráneo y post cráneo, centrado en la va- riabilidad de la calidad de vida biológica en los grupos atacameños durante las distintas fases de su desarrollo prehispánico. Un gran número de estos trabajos sigue las ideas investigadas por Le Paige y Costa acerca de la relación de los atacameños con grupos foráneos, y en particular con el Estado altiplánico de Tiwanaku (TorresRouff 2002, 2008; Costa et al. 2004; Knudson 2004, 2007, 2008; Lessa y Mendonça de Souza 2004, 2006; Knudson y Price 2007; Knudson y Blom 2009). Dentro de los trabajos que investigaban estilo de vida están aquellos que se centran en dieta y nutrición (Neves y Costa 1998; Neves et al. 1999; Costa et al. 2004), y en tensión social (Lessa 1995, 2005; Costa et al. 1998; Lessa y Mendonça de Souza 2003-2004, 2004, 2006, 2007, 2009; Torres-Rouff et al. 2005; Neves et al. 2006; Torres-Rouff y Costa 2006), además de otros trabajos con temas puntuales (Núñez et al. 1998; Torres-Rouff 2003; Marsteller et al. 2011). Finalmente, en la última década, se han iniciado nuevas líneas de investigación sobre temas de adaptación al medio (Weinstein 2001, 2005, 2007) y movilidad humana (Knudson 2007, 2009; Torres-Rouff y Knudson 2007; Costa et al. 2009; Knudson y TorresRouff 2009). Estos trabajos traen consigo importantes avances tecnológicos y metodológicos, siendo los más notables los análisis de isótopos incorporados en los trabajos de Knudson y el análisis de ADN de patógenos en muestras prehispánicas locales (Núñez et al. 1998; Costa et al. 2009).

Entrando a la segunda década del s. XXI, la colección osteológica Le Paige continúa siendo de gran interés para académicos nacionales e internacionales. En la actualidad se encuentran en ejecución cuatro proyectos de investigación bioantropológica, financiados por importantes instituciones científicas entre las que se cuentan FONDECYT, CONICYT y National Science Foundation.

\section{* Estado de conservación de la colección osteológICA LE PAIGE}

A lo largo de los últimos cinco años, hemos realizado un inventario y diagnóstico completo de los restos osteológicos depositados en el IIAM. Nuestra motivación para realizar la catalogación fue efectuar un diagnóstico de la colección y así determinar cuáles elementos se encontraban en mal estado de conservación y/o con problemas 
de registro. De esta manera, registramos la totalidad de los restos óseos depositados en el IIAM, identificándolos con un número de tumba/catálogo y clasificándolos como bueno, regular o malo, de acuerdo a su estado de preservación. Los cráneos y cuerpos clasificados como en "buen estado de conservación" son todos aquellos que presentan registros claros, que corresponden a la numeración de las tumbas dada por Le Paige, y por lo tanto, están en condiciones de ser estudiados científicamente. Los cráneos clasificados como en "estado regular de conservación" son aquellos que, aunque no presenten problemas en su registro e identificación, se encuentran fragmentados y necesitan de intervenciones menores para alcanzar un estado de conservación aceptable. Es decir, podrían ser incluidos en estudios científicos, dependiendo de los objetivos. Finalmente, los cráneos y cuerpos en "mal estado de conservación" son aquellos que no cuentan con una numeración confiable, de manera que no pueden ser asignados con seguridad a uno de los cementerios excavados por Le Paige, o bien, requieren de una gran inversión de tiempo para estabilizar su estado de conservación. Son, por lo tanto, materiales que no están disponibles para estudios científicos y que, en la mayoría de los casos, jamás lo estarán por la imposibilidad de recuperar su contexto y numeración original. A la fecha, de las 5482 tumbas registradas por Le Paige, encontramos solamente $2343(42,7 \%)$ cráneos o cuerpos en buen estado de conservación y 723 (13,2\%) cráneos o cuerpos en estado regular de conservación. El resto está representado por $612(11,4 \%)$ restos en mal estado de conservación y 696 (13\%) cráneos con numeración duplicada, cráneos sin numeración, o desaparecidos. Por lo tanto, actualmente, solo un poco más de la mitad de la colección original excavada por Le Paige, está disponible para estudios científicos.

Queremos destacar aquí algunas causas relacionadas con la pérdida de la contextualización y de la mala conservación detectadas en la colección en estos últimos cinco años. La más grave, a nuestro parecer, es la numeración duplicada de 696 cráneos. Aun cuando Le Paige generó una numeración secuencial para todas las tumbas excavadas en la cuenca del Salar de Atacama y del Loa, de vez en cuando se equivocó en la secuencia de la numeración, por lo que ciertos sitios presentan números repetidos, algunos de ellos anotados como "bis". Sin embargo, esto es solo una minoría de las tumbas excavadas por Le Paige, y no es el mayor problema que observamos en la actualidad. Muchos de los cráneos reportados aquí presentan números duplicados debido a que en algún momento posterior a la muerte de Le Paige, fueron vueltos a marcar, algunos de manera equivocada, y que no corresponden a los duplicados del sacerdote. Con eso, no solo se descontextualizaron los cráneos equivocados, sino también los cráneos correctamente numerados, debido a que en la mayoría de los casos es difícil o casi imposible saber cuál es el cráneo original y cual recibió la numeración equivocada. Le Paige tomó medidas de la gran mayoría de los cráneos que excavó, así que en el futuro creemos que será posible solucionar parte de los problemas de ambigüedad de registro, comparando las medidas de los cráneos con números duplicados. A estos casos, se suma una parte significativa de la colección, que son los cráneos cuya numeración se perdió en las últimas décadas y que no pueden ser actualmente asignados a ningún cementerio.

Se suma al problema de numeración de los cráneos, el hecho de que en las últimas cuatro décadas parte de la colección sufrió un deterioro progresivo de su estado de conservación debido a su abandono o desaparición. El ejemplo más emblemático se ve en la colección de cráneos de Toconao Oriente, un cementerio en el cual Le Paige registró 545 tumbas, de las cuales solamente 145 presentan un estado de conservación bueno o regular. Parte de esta colección fue encontrada en el año 2008, en cajas con cráneos muy fragmentados y envueltos en diarios de 1968 y 1971.

Aquí no abordaremos los problemas de las mandíbulas que fueron separadas de los cráneos, ya que todavía no se ha cuantificado la cantidad de cráneos sin mandíbulas o la cantidad de mandíbulas que están numeradas y que en el futuro podrán ser asociadas a sus respectivos cráneos. Sin embargo, se debe aclarar que en este momento, gran parte de las mandíbulas se encuentran separadas de sus cráneos y solamente una parte de ellas cuenta con algún tipo de numeración que permitirá reasignarlas.

Finalmente, existe en la actualidad una cantidad desconocida de cráneos que fueron donados por Le Paige o sus seguidores a museos internacionales incluyendo el reino Belga, el Museo de Historia Natural de Nueva York y el Museo de América en Madrid. Por no existir registro de 
estas transacciones, es imposible estimar la cantidad real de cráneos que se encuentran en instituciones extranjeras.

Los problemas que hemos comentado no tienen como objetivo condenar prácticas particulares del pasado, pero nos parece necesario explicar sucintamente las razones por las cuales la colección presenta el estado actual de conservación. Queremos, en este trabajo, establecer un diagnóstico inicial que será usado como punto de partida de los trabajos de recontextualización de lo que sea posible recuperar. Además, como ya hemos mencionado, nuestro objetivo es generar un catálogo contextualizado que sirva de referencia para profesionales interesados en incluir esta colección en sus investigaciones y, de esta forma, promover su utilidad científica. Por ello, hemos elaborado la Tabla 1 que presenta los cementerios excavados por Le Paige, la cantidad de tumbas informadas por él y los cráneos o cuerpos que se encuentran en estado de conservación bueno o regular.

A continuación, la Tabla 2 presenta un listado de los sitios que fueron excavados por Le Paige cuyos individuos no fueron identificados dentro de la colección. La mayoría de estos se refieren a excavaciones puntuales o rescates arqueológicos que recuperaron un número pequeño de individuos. Aquí no hacemos la distinción entre cuerpos momificados y cráneos individuales, aunque la cantidad total de cuerpos identificados con número de inventario evidente e incuestionable es muy pequeña $(n=110)$. El trabajo de Jacobs (1986) presenta una descripción e inventario detallado de los cuerpos momificados, y sirve de referencia para quienes se interesen por el tópico.

En cada sitio se observa una situación variable de conservación del material. De los 23 cementerios mayores excavados por Le Paige, actualmente tres presentan menos de 50\% de las tumbas excavadas en buen o regular estado de conservación (p.e., Sequitor Alambrado, Tchaputchayna y Toconao Oriente); de los demás, 16 presentan más de $25 \%$ de sus individuos sin las condiciones necesarias para su análisis. Con el fin de dar una idea acerca de la representatividad geográfica de los cementerios, en la Figura i presentamos la ubicación de esos sitios mayores. El producto final de este inventario será un catálogo completo con el listado de los individuos ubicados, su sexo y edad, además de informaciones osteológicas básicas a su respecto. Este inventario final se encontrará disponible, en el IIAM, para los investigadores interesados en trabajar con estas colecciones.

\section{* Contexto cronológico de los cementerios EXCAVADOS POR LE PAIGE}

Para refinar la contextualización cronológica de la colección Le Paige, nuestro trabajo de inventario y análisis de sus condiciones de preservación incluyó la selección de muestras osteológicas de los cementerios más representativos, para datarlas por radiocarbón AMS. Nuestra intención es presentar junto con el estado de conservación de la colección, un contexto cronológico directo de las ocupaciones humanas representadas por los cementerios más numerosos excavados por Le Paige. De esta manera, esperamos poder complementar el marco cronológico existente para las series cerámicas (Tarragó 1968; Berenguer et al. 1986, 1988; Uribe 2002), aportando con una mayor comprensión de los procesos de ocupación de la región atacameña en el período comprendido entre 2250 y $500 \mathrm{AP}$.

En la Tabla 3, presentamos 50 dataciones radiocarbónicas (AMS- $\mathrm{C}^{14}$ ) para 21 cementerios excavados por Le Paige. Las dataciones fueron realizadas en dos laboratorios de Estados Unidos: Beta Analytic y el University of Arizona Accelerator Mass Spectrometry Laboratory. Ambos laboratorios son prestigiados centros de dataciones radiocarbónicas, con estrictos protocolos de control de calidad. Las dataciones fueron realizadas sobre la fracción orgánica (colágeno) de muestras de tejidos óseos extraídos de las colecciones de cráneos del IIAM, siguiendo los protocolos de extracción de cada laboratorio. Fueron seleccionados para ser datados solamente cráneos que no tuviesen ningún problema de identificación y contextualización. Elegimos datar los cementerios más representativos numéricamente excavados por Le Paige que no contaban todavía con un marco cronológico absoluto. Sin embargo, en algunos casos, para corroborar las dataciones de termoluminiscencia sobre cerámicas, se dataron algunos individuos de sitios considerados por Berenguer y colaboradores (1986). Para todas las dataciones, fueron calculadas fechas calibradas usando la curva SHCALo4 en el programa Calib 6.0 (Stuiver y Reimer 1993; McCormac et al. 2004). 


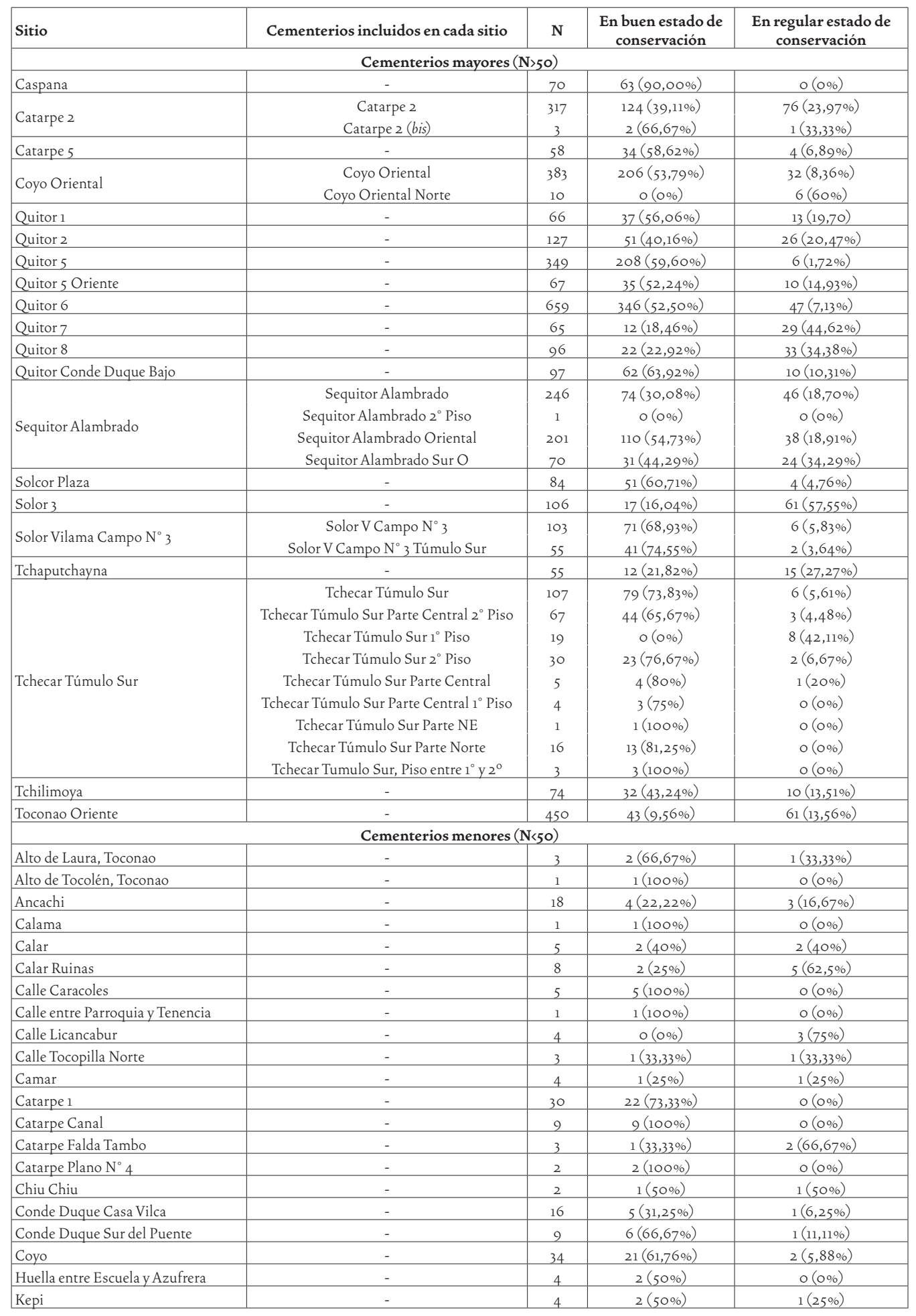

Tabla 1. Cementerios excavados por Le Paige ordenados de acuerdo a su tamaño y alfabéticamente; número de tumbas informadas para cada cementerio y cantidad de individuos (cráneos o cuerpos) encontrados actualmente ( $\mathrm{N=3205}$ ). 
Mark Hubbe, Macarena Oviedo, Christina Torres-Rouff

\begin{tabular}{|c|c|c|c|c|}
\hline La Cruz Larache & - & 13 & $2(15,38 \%)$ & $3(23,08 \%)$ \\
\hline Larache $1^{\circ}$ Piso & - & 16 & $10(62,5 \%)$ & $3(18,75 \%)$ \\
\hline Larache Acequia Huerto Reales & - & 1 & $1(100 \%)$ & $\mathrm{O}(\mathrm{o} \%)$ \\
\hline Larache Callejón Reales & $\begin{array}{c}\text { Larache Callejón } \\
\text { Larache Callejón } 1^{\circ} \text { Piso } \\
\text { Larache Callejón } 2^{\circ} \text { Piso } \\
\text { Larache Callejón Reales } \\
\text { Larache Capa Inferior } 2^{\circ} \text { Piso }\end{array}$ & $\begin{array}{c}1 \\
14 \\
21 \\
15 \\
1\end{array}$ & $\begin{array}{c}0(0 \%) \\
6(42,86 \%) \\
9(42,86 \%) \\
5(33,33 \%) \\
1(100 \%)\end{array}$ & $\begin{array}{l}0(0 \%) \\
1(7,14 \%) \\
2(9,52 \%) \\
2(13,33 \%) \\
0(0 \%)\end{array}$ \\
\hline Lava Oriental & - & 2 & $1(50 \%)$ & $\mathrm{o}(\mathrm{O} \%)$ \\
\hline Patio Casa Parroquial & - & 1 & $\mathrm{o}(\mathrm{0} \%)$ & $1(100 \%)$ \\
\hline Peine & - & 14 & $3(21,43 \%)$ & $9(64,29 \%)$ \\
\hline Poconche & - & 5 & $2(40 \%)$ & $2(40 \%)$ \\
\hline Poconche Túmulo Sur & - & 5 & $2(40 \%)$ & $1(20 \%)$ \\
\hline Quitor Conde Duque 3 Más Abajo & - & 2 & $1(50 \%)$ & $\mathrm{o}(0 \%)$ \\
\hline Quitor I Conde Duque Arriba & - & 2 & $\mathrm{o}(\mathrm{o} \%)$ & $1(50 \%)$ \\
\hline Quitor 2 Conde Duque Bajo & - & 1 & $1(100 \%)$ & $\mathrm{O}(\mathrm{O} \%)$ \\
\hline Quitor 3 (bis) & - & 3 & $2(66,67 \%)$ & $\mathrm{o}(0 \%)$ \\
\hline Quitor 4 Conde Duque & $\begin{array}{l}\text { Quitor } 4 \text { (C.D.) Cementerio más al Sur } \\
\text { Quitor } 4 \text { Conde Duque Norte Puente }\end{array}$ & $\begin{array}{l}21 \\
25\end{array}$ & $\begin{array}{c}10(47,62 \%) \\
16(64 \%)\end{array}$ & $\begin{array}{c}1(4,76 \%) \\
3(12 \%)\end{array}$ \\
\hline Quitor 5 Sur Oriente & - & 37 & $11(29,73 \%)$ & $4(10,8 \%)$ \\
\hline Quitor 9 & - & 19 & $11(57,89 \%)$ & $7(36,84 \%)$ \\
\hline Quitor 10 & - & 4 & $1(25 \%)$ & $1(25 \%)$ \\
\hline Quitor 11 & - & 3 & $1(33,33 \%)$ & $\mathrm{o}(\mathrm{o} \%)$ \\
\hline Quitor Casa Ysidora Mondaca & - & 2 & $2(100 \%)$ & $\mathrm{o}(\mathrm{o} \%)$ \\
\hline Quitor Cerca del Pucara & - & 1 & $1(100 \%)$ & $\mathrm{o}(\mathrm{O} \%)$ \\
\hline Quitor Conde Duque & $\begin{array}{l}\text { Quitor Conde Duque } \\
\text { Quitor Conde Duque 2 Cementerio } \\
\text { Quitor Conde Duque } 3 \text { más Abajo } \\
\text { Quitor Conde Duque Cementerio Bajo } \\
\text { Quitor Conde Duque más Abajo }\end{array}$ & $\begin{array}{l}32 \\
3 \\
2 \\
8 \\
4\end{array}$ & $\begin{array}{c}14(43,75 \%) \\
1(33,33 \%) \\
1(50 \%) \\
1(12,5 \%) \\
0(0 \%)\end{array}$ & $\begin{array}{c}2(6,25 \%) \\
0(0 \%) \\
0(0 \%) \\
1(12,5 \%) \\
2(50 \%) \\
\end{array}$ \\
\hline Quitor Silo & - & 24 & $11(45,83 \%)$ & $1(4,17 \%)$ \\
\hline Quitor & - & 3 & $3(100 \%)$ & $\mathrm{o}(\mathrm{o} \%)$ \\
\hline Rio Grande (San Juan) & - & 5 & $4(80 \%)$ & $\mathrm{O}(\mathrm{o} \%)$ \\
\hline San Pedro de Atacama, Casa F. & - & 2 & $\mathrm{O}(\mathrm{O} \%)$ & $1(50 \%)$ \\
\hline San Pedro, Matadero & - & 1 & $1(100 \%)$ & $\mathrm{o}(\mathrm{o} \%)$ \\
\hline San Pedro, Pueblo & - & 6 & $5(83,33 \%)$ & $\mathrm{o}(\mathrm{o} \%)$ \\
\hline Sequitor Occidental & - & 1 & $1(100 \%)$ & $\mathrm{o}(\mathrm{O} \%)$ \\
\hline Sequitor Oriental & - & 5 & $5(100 \%)$ & $\mathrm{o}(0 \%)$ \\
\hline Sequitor & - & 3 & $1(33,33 \%)$ & $1(33,33 \%)$ \\
\hline Sequitor Tchasquir & - & 1 & $1(100 \%)$ & $\mathrm{O}(\mathrm{o} \%)$ \\
\hline Socaire Pueblo & - & 5 & $2(40 \%)$ & $1(20 \%)$ \\
\hline Solcor Casa L. Sandon & - & 1 & $1(100 \%)$ & $\mathrm{o}(\mathrm{o} \%)$ \\
\hline Solcor Nueva Población & - & 32 & $1(3,13 \%)$ & $21(65,63 \%)$ \\
\hline Solcor Plaza Oriental & $\begin{array}{c}\text { Solcor Plaza Oriental } \\
\text { Solcor Plaza Oriental (bis) }\end{array}$ & $\begin{array}{c}17 \\
1\end{array}$ & $\begin{array}{c}9(52,94 \%) \\
1(100 \%)\end{array}$ & $\begin{array}{c}3(17,65 \%) \\
0(0 \%)\end{array}$ \\
\hline Solcor & - & 3 & $3(100 \%)$ & $\mathrm{o}(0 \%)$ \\
\hline Solor 3 Túmulo Norte & $\begin{array}{c}\text { Solor } 3 \text { Túmulo Norte } 1^{\circ} \text { Piso } \\
\text { Solor } 3 \text { Túmulo Norte } \\
\text { Solor } 3 \text { Oriente del Túmulo Norte }\end{array}$ & $\begin{array}{c}19 \\
1 \\
2\end{array}$ & $\begin{array}{c}\mathrm{O}(\mathrm{O} \%) \\
1(100 \%) \\
\mathrm{O}(0 \%)\end{array}$ & $\begin{array}{l}1(5,27 \%) \\
0(0 \%) \\
0(0 \%)\end{array}$ \\
\hline Solor 3 Túmulo Sur & - & 18 & $14(77,78 \%)$ & $2(11,11 \%)$ \\
\hline Solor 4 & - & 5 & $2(40 \%)$ & $\mathrm{o}(\mathrm{o} \%)$ \\
\hline Solor 5 Norte & - & 3 & $1(33,33 \%)$ & $\mathrm{o}(\mathrm{o} \%)$ \\
\hline Solor 6 & - & 13 & $3(23,08 \%)$ & $4(30,77 \%)$ \\
\hline Solor Campo $\mathrm{N}^{\circ} 3$ & $\begin{array}{l}\text { Solor Campo } \mathrm{N}^{\circ} 3 \text { Lado Occidental } \\
\text { Solor Campo } \mathrm{N}^{\circ} 3 \text { Túmulo Norte } \\
\text { Solor Campo } \mathrm{N}^{\circ} 3 \text { Túmulo Sur } \\
\text { Solor Campo } \mathrm{N}^{\circ} 3{\text { Túmulo Norte } 1^{\circ} \text { Piso }}^{\text {Solor V }\left(\text { Campo }_{3}\right)}\end{array}$ & $\begin{array}{c}7 \\
40 \\
23 \\
1 \\
2 \\
\end{array}$ & $\begin{array}{c}2(28,57 \%) \\
22(55 \%) \\
14(60,87 \%) \\
0(0 \%) \\
0(0 \%)\end{array}$ & $\begin{array}{c}2(28,57 \%) \\
7(17,5 \%) \\
1(4,35 \%) \\
0(0 \%) \\
0(0 \%) \\
\end{array}$ \\
\hline Solor V Campo $\mathrm{N}^{\circ} 1$ & $-{ }^{2}$ & 2 & $2(100 \%)$ & $\mathrm{O}(\mathrm{O} \%)$ \\
\hline Solor V Campo $\mathrm{N}^{\circ} 2$ & - & 4 & $1(25 \%)$ & $2(50 \%)$ \\
\hline Solor V Campo $\mathrm{N}^{\circ} 3$ Túmulo Norte & - & 3 & $2(66,67 \%)$ & $1(33,33 \%)$ \\
\hline Solor V Campo $\mathrm{N}^{\circ} 4$ & - & 30 & $19(63,33 \%)$ & $3(10 \%)$ \\
\hline
\end{tabular}

Continuación Tabla 1.

$36 \quad \begin{aligned} & \mathrm{N}^{\circ} 41 / 2011 \\ & \text { EstUdios ATACAMEÑos } \\ & \text { Arqueología y Antropología Surandinas }\end{aligned}$ 


\begin{tabular}{|c|c|c|c|c|}
\hline Solor V Campo $\mathrm{N}^{\circ} 5$ & - & 6 & $3(50 \%)$ & $2(33,33 \%)$ \\
\hline Solor V Campo $\mathrm{N}^{\circ} 6$ & - & 26 & $13(50 \%)$ & $2(7,69 \%)$ \\
\hline Talabre, Quebrada de Ama & - & 1 & $1(100 \%)$ & $\mathrm{o}(0 \%)$ \\
\hline Tchapuraqua Toconao & - & 1 & $0(0 \%)$ & $1(100 \%)$ \\
\hline Tchaputchayna Cementerio & - & 13 & $1(7,69 \%)$ & $3(23,08 \%)$ \\
\hline Tchecar Túmulo Norte & - & 6 & $5(87,50 \%)$ & $1(12,50 \%)$ \\
\hline Tchecar Sur & - & 3 & $2(66,67 \%)$ & $\circ(\mathrm{o} \%)$ \\
\hline Tilomonte & - & 32 & $17(53,13 \%)$ & $7(21,88 \%)$ \\
\hline Tilomonte 1 & - & 6 & $6(100 \%)$ & $\mathrm{o}(0 \%)$ \\
\hline Tilomonte 1A & - & 1 & $1(100 \%)$ & $\mathrm{o}(0 \%)$ \\
\hline Tilomonte 2 & - & 1 & $1(100 \%)$ & $\mathrm{O}(0 \%)$ \\
\hline Tilomonte 5 & - & 2 & $2(100 \%)$ & $\mathrm{o}(0 \%)$ \\
\hline Tilomonte Algarrobo & - & 1 & $1(100 \%)$ & $\mathrm{o}(0 \%)$ \\
\hline Tilomonte Sur & - & 25 & $18(72 \%)$ & $4(16 \%)$ \\
\hline Toco, San Pedro & - & 4 & $3(75,00 \%)$ & $\mathrm{o}(0 \%)$ \\
\hline Toconao & - & 10 & $7(70,00 \%)$ & $3(30,00 \%)$ \\
\hline Toconce & - & 30 & $25(83,33 \%)$ & $1(3,33 \%)$ \\
\hline Tulor Algarrobo & - & 5 & $1(20 \%)$ & $1(20 \%)$ \\
\hline Tulor Ruinas & $\begin{array}{c}\text { Tulor Pueblo Ruinas } \\
\text { Tulor Ruinas } 1 \\
\text { Tulor } \\
\end{array}$ & $\begin{array}{l}8 \\
9 \\
2\end{array}$ & $\begin{array}{l}4(50 \%) \\
0(0 \%) \\
1(50 \%) \\
\end{array}$ & $\begin{array}{c}2(25 \%) \\
4(44,44 \%) \\
1(50 \%) \\
\end{array}$ \\
\hline Turi & - & 1 & $1(100 \%)$ & $\mathrm{o}(0 \%)$ \\
\hline Vilama & - & 13 & $9(69,23 \%)$ & $1(7,69 \%)$ \\
\hline Vilama Huella & - & 19 & $15(78,95 \%)$ & $2(10,53 \%)$ \\
\hline Yaye 1 & - & 15 & $7(46,67 \%)$ & $3(20 \%)$ \\
\hline Yaye 2 & - & 87 & $57(65,52 \%)$ & $7(8,05 \%)$ \\
\hline Yaye 3 en la curva & - & 32 & $20(62,5 \%)$ & $6(18,75 \%)$ \\
\hline Yaye 4 & $\begin{array}{c}\text { Yaye } 4 \\
\text { Yaye } 4 \text { (bis) }\end{array}$ & $\begin{array}{c}8 \\
20\end{array}$ & $\begin{array}{c}5(62,5 \%) \\
13(65 \%)\end{array}$ & $\begin{array}{c}1(12,5 \%) \\
1(5 \%)\end{array}$ \\
\hline Yaye Orilla Occidental & $\begin{array}{l}\text { Yaye Orilla Occidental } \\
\text { Yaye Orilla Occidental } 1\end{array}$ & $\begin{array}{c}49 \\
3\end{array}$ & $\begin{array}{l}36(73,47 \%) \\
2(66,67 \%)\end{array}$ & $\begin{array}{c}4(8,16 \%) \\
0(0 \%)\end{array}$ \\
\hline Zapar Toconao & - & 1 & $\mathrm{o}(0 \%)$ & $1(100 \%)$ \\
\hline
\end{tabular}

Continuación Tabla 1.

La Figura 2 muestra la dispersión de las fechas para cada sitio. En general, se observa que la gran mayoría de los cementerios fueron ocupados por largos períodos, más de cinco siglos en algunos casos, por generaciones consecutivas. Eso corrobora la idea de que la ocupación humana en los oasis fue estable y organizada en torno a lugares sagrados, en este caso, cementerios (Berenguer et al. 1986, 1988; Llagostera y Costa 1999).

Las nuevas dataciones que presentamos abarcan mayoritariamente una larga ocupación de los oasis, pero contribuyen poco a los primeros momentos de su poblamiento (fases Tilocalar 3450-2250 AP y Toconao 2250-1850 AP). Sin embargo, obtuvimos fechas relativamente tempranas para Quitor 2 y Solor 3, que favorecen la idea de que la ocupación humana de los ayllu empezó a ser más intensa durante la fase Sequitor (1850-1550 AP), en los primeros siglos de nuestra era. Tal como lo han propuesto Llagostera y Costa (1999), nuestros datos muestran una ocupación humana dis-

\begin{tabular}{|l|c|l|c|}
\hline Cementerio & N & Cementerio & N \\
\hline Casa Parroquial & 1 & Sala de Catequismo & 1 \\
Catarpe Norte & 1 & Sequitor & 1 \\
Catarpe Plano $\mathrm{N}^{\circ} 3$ & 3 & Sequitor Plaza & 5 \\
Club Atacama & 1 & Sequitor Tchasquir & 5 \\
Cucuter & 1 & Solcor NE & 4 \\
Frente Casa Parroquial & 1 & Solcor Norte del Ayllu & 2 \\
Huerto Casa Terrazas & 2 & Solor Ayllu & 1 \\
Larache Antiguo Canal & 1 & Solor Campo $\mathrm{N}^{\circ} 2$ & 2 \\
Quillagua & 3 & Solor Campo $\mathrm{N}^{\circ} 4$ & 2 \\
Quitor Norte de la Turbina & 1 & Tchecar & 1 \\
\hline
\end{tabular}

Tabla 2. Listado de cementerios excavados por Le Paige que no fueron identificados en el inventario de la colección.

persa en gran parte de los ayllu al inicio de la fase Quitor (1550-1250 AP). Las fechas presentadas aquí corroboran la sugerencia de esos autores, acerca de que esta 
Mark Hubbe, Macarena Oviedo, Christina Torres-Rouff

\begin{tabular}{|c|c|c|c|c|}
\hline Cementerio & Tumba & $\mathrm{N}^{\circ}$ de Laboratorio & $\begin{array}{c}\text { Fechado absoluto } \\
\text { Años AP }\end{array}$ & $\begin{array}{c}\text { Fecha calibrada (2 sigmas) } \\
\text { Años calendáricos) }\end{array}$ \\
\hline Caspana & 32 & Beta-263464 & $660 \pm 40$ & $1294-1403$ DC \\
\hline Caspana & 43 & Beta-263465 & $610 \pm 40$ & $1304-1435$ DC \\
\hline Caspana & 45 & Beta-263466 & $670 \pm 40$ & $1291-1400$ DC \\
\hline Catarpe 1 & 294 & AA87009 & $752 \pm 43$ & $1225^{-13} 88 \mathrm{DC}$ \\
\hline Catarpe 1 & 2397 & AA87010 & $689 \pm 43$ & $1284-1396 \mathrm{DC}$ \\
\hline Catarpe 2 & 1753 & Beta-251747 & $1220 \pm 40$ & $734-983$ DC \\
\hline Catarpe 2 & 1786 & Beta-251748 & $750 \pm 40$ & $1228-1387$ DC \\
\hline Catarpe 2 & 1801 & Beta-251749 & $1030 \pm 40$ & $992-1151 \mathrm{DC}$ \\
\hline Catarpe 2 & 1850 & Beta-251750 & $770 \pm 40$ & $1221-1382 \mathrm{DC}$ \\
\hline Catarpe 5 & 2385 & AA87011 & $1083 \pm 47$ & $895-1145$ DC \\
\hline Catarpe 5 & 2392 & AA87012 & $1123 \pm 44$ & 879-1039 DC \\
\hline Larache Callejón Real & 5056 & $\mathrm{AA} 87013$ & $1667 \pm 45$ & $337-562$ DC \\
\hline Quitor 1 & 3487 & AA87014 & $956 \pm 44$ & $1032-1211$ DC \\
\hline Quitor 2 & 3684 & AA87015 & $1491 \pm 46$ & $539-669 \mathrm{DC}$ \\
\hline Quitor 2 & 3716 & Beta-251751 & $1520 \pm 40$ & $465-659 \mathrm{DC}$ \\
\hline Quitor 2 & 3770 & AA87016 & $1696 \pm 46$ & $259-540 \mathrm{DC}$ \\
\hline Quitor 2 & 3783 & Beta-251752 & $1310 \pm 40$ & $671-874$ DC \\
\hline Quitor 5 & 1921 & AA87017 & $1164 \pm 44$ & 782-1019 DC \\
\hline Quitor 5 & 2009 & AA87018 & $1511 \pm 46$ & $443-664$ DC \\
\hline Quitor 5 & 2179 & AA87019 & $1338 \pm 45$ & $656-865 \mathrm{DC}$ \\
\hline Quitor 5 & 3394 & AA87020 & $1623 \pm 46$ & 401-599 DC \\
\hline Quitor 6 & 2529 & Beta-263467 & $1050 \pm 40$ & $982-1150$ DC \\
\hline Quitor 6 & 2588 & Beta-263468 & $1290 \pm 40$ & 681-886 DC \\
\hline Quitor 6 & 2928 & Beta-263469 & $1180 \pm 40$ & $780-992 \mathrm{DC}$ \\
\hline Quitor 6 & 3633 & Beta-263470 & $1490 \pm 40$ & $551-662 \mathrm{DC}$ \\
\hline Quitor 8 & 3145 & Beta-251753 & $1510 \pm 40$ & $538-659$ DC \\
\hline Quitor 8 & 3226 & Beta-251754 & $1450 \pm 40$ & $566-757 \mathrm{DC}$ \\
\hline Quitor 9 & 3251 & $\mathrm{AA} 87022$ & $1068 \pm 44$ & 899-1149 DC \\
\hline Sequitor Alambrado & 533 & Beta-263471 & $1430 \pm 40$ & $591-768 \mathrm{DC}$ \\
\hline Sequitor Alambrado & 1043 & Beta-263472 & $1680 \pm 40$ & $265-544 \mathrm{DC}$ \\
\hline Sequitor Alambrado & 1062 & Beta-251745 & $1600 \pm 40$ & $422-602 \mathrm{DC}$ \\
\hline Sequitor Alambrado & 1068 & Beta-251746 & $1680 \pm 40$ & $265-544 \mathrm{DC}$ \\
\hline Solcor Plaza & 759 & $\mathrm{AA} 87023$ & $1535 \pm 45$ & $436-654 \mathrm{DC}$ \\
\hline Solcor Plaza & 1241 & $\mathrm{AA} 87024$ & $987 \pm 44$ & $1019-1201$ DC \\
\hline Solor 3 & 983 & $\mathrm{AA} 87026$ & $1616 \pm 46$ & 406-602 DC \\
\hline Solor 3 & 991 & $\mathrm{AA} 87027$ & $1859 \pm 47$ & 83-375 DC \\
\hline Solor Vilama 3 & 265 & AA87028 & $1076 \pm 45$ & 896-1147 DC \\
\hline Solor Vilama 3 & 308 & AA87029 & $1125 \pm 45$ & 871-1041 DC \\
\hline Tchecar Túmulo Sur & 650 & Beta-263476 & $1090 \pm 40$ & $895-1132 \mathrm{DC}$ \\
\hline Tchecar Túmulo Sur & 806 & Beta-263478 & $960 \pm 40$ & $1033-1206$ DC \\
\hline Tchecar Túmulo Sur & 824 & Beta-263479 & $1240 \pm 40$ & 711-971 DC \\
\hline Toconce & 78 & Beta-263473 & $760 \pm 40$ & $1224-1384$ DC \\
\hline Toconce & 80 & Beta-263474 & $490 \pm 40$ & $1405^{-1610}$ DC \\
\hline Toconce & 97 & Beta-263475 & $920 \pm 40$ & $1042-1260$ DC \\
\hline Yaye 1 & 5494 & Beta-251755 & $920 \pm 40$ & $1042-1260 \mathrm{DC}$ \\
\hline Yaye 1 & 5498 & Beta-251756 & $1100 \pm 40$ & 892-1102 DC \\
\hline Yaye 2 & 3309 & Beta-251757 & $1300 \pm 40$ & $667-881 \mathrm{DC}$ \\
\hline Yaye 2 & 3417 & Beta-251758 & $1040 \pm 40$ & $988-1150$ DC \\
\hline Yaye 3 & 1573 & Beta-251759 & $1180 \pm 40$ & $780-992 \mathrm{DC}$ \\
\hline Yaye 4 & 1545 & Beta-251760 & $1170 \pm 40$ & $782-1016 \mathrm{DC}$ \\
\hline
\end{tabular}

Tabla 3. Dataciones radiocarbónicas (AMS- ${ }^{14}$ ) de 50 muestras óseas provenientes de 21 cementerios excavados por Le Paige en las cuencas del Salar del Atacama y del río Loa.

\footnotetext{
\begin{tabular}{l|l}
38 & $\begin{array}{l}\mathrm{N}^{\circ} 41 / 2011 \\
\text { ESTUdios ATACAMEÑOS } \\
\text { Arqueología y Antropología Surandinas }\end{array}$
\end{tabular}
} 


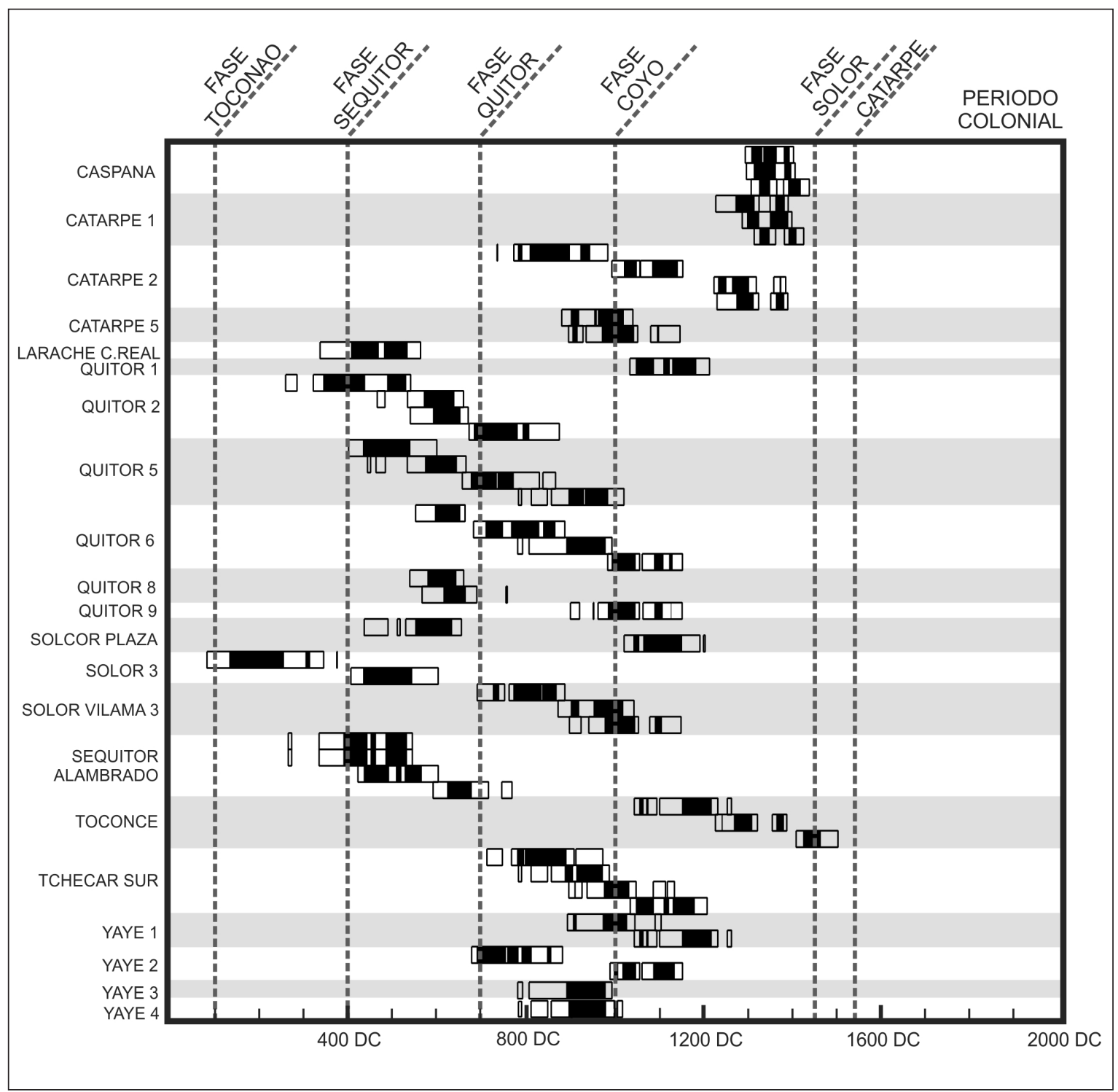

Figura 2. Fechas calibradas (SHCALO4). Las celdas oscuras indican el 68\% de probabilidad de calibración; las celdas claras indican el $95 \%$ de probabilidad de calibración.

ocupación tuvo mayor fuerza en los ayllu centrales de San Pedro de Atacama, como Quitor, Larache, Solcor, Solor y Sequitor (ver Figura 1).

Los datos obtenidos señalan que la expansión hacia los demás ayllu (Catarpe, Solor-Vilama, Tchecar y Yaye) no ocurre hasta fines de la fase Coyo (1250-950 AP), casi coincidiendo con el retiro de la influencia de Tiwanaku de la zona. La ocupación de esos ayllu durante la fase Solor
(950-500 AP) del período Intermedio Tardío se ve claramente asociada a la reestructuración de la vida en los oasis tras el vacío dejado por Tiwanaku y al inicio de un período de sequía intenso en la región (Schiapacasse et al. 1989; Nielsen 2002). La mayor ocupación de Catarpe y Solor-Vilama en ese momento sugiere que estos cambios significativos en el estilo de vida local están asociados con un esfuerzo por ocupar y posiblemente controlar las dos principales fuentes de agua de los oasis, los ríos 
Vilama y San Pedro (Adán 1999). De manera similar, se observa el surgimiento de cementerios en Yaye, un ayllu cercano al trayecto actual del río San Pedro, y que por lo tanto apoya la idea de que la disminución de la disponibilidad de agua en la región promovió el acercamiento de los grupos locales a los ríos. Nuestros datos sugieren que esto empezó en un momento anterior a lo propuesto por Llagostera y Costa (1999).

Finalmente, con excepción de una fecha de Catarpe 2 (Tabla 1 y Figura 2), ninguna otra cruza el período de influencia incaica (fase Catarpe 500-400 AP), apoyando la idea de que el centro administrativo incaico, instalado en Catarpe, y adyacente al camino inca (Lynch 1993; Llagostera y Costa 1999), se benefició de una estructura preexistente, centrando su administración en un ayllu que ya se encontraba ocupado por lo menos 500 años antes de su llegada (Lynch 1993; Adán 1999; Alden et al. 2006; Acuto 2008).

\section{$*$ Comentarios finales}

Con el mismo objetivo que se planteó Le Paige en su primer trabajo en Estudios Atacameños en 1973, esperamos que nuestro aporte destaque la importancia científica que tiene la colección osteológica del IIAM. Hemos documentado su estado actual de conservación y presentado nuevos datos cronológicos que serán útiles para futuras investigaciones. Numerosos trabajos ya publicados sobre la colección han documentado muchos otros detalles y contextos biológicos sobre los restos humanos excavados en el desierto de Atacama desde los años 1950. Esta co- lección sirve como una fuente de conocimiento incomparable sobre la vida de los antiguos atacameños.

Esperamos que los datos que hemos proporcionado promuevan a una serie de nuevas investigaciones tanto arqueológicas como bioantropológicas que tomen como referencia a esta colección. Las fechas de los cementerios sugieren una larga ocupación de la mayoría de los oasis atacameños, lo cual permitirá la realización de análisis bioarqueológicos acerca de los efectos de los grandes cambios ocurridos en los Andes Centro Sur. Además, surgen numerosas posibilidades para investigaciones diacrónicas sobre la población atacameña.

En suma, los restos humanos albergados en el IIAM constituyen una de las mayores y más importantes colecciones osteológicas de América Latina y ahora cuentan con un marco geográfico y cronológico que facilitará muchas más décadas de investigación científica.

Agradecimientos Esta contribución es dedicada al padre Gustavo Le Paige, por su trabajo metódico y su esfuerzo en comprender la prehistoria atacameña. Este trabajo es parte de los Proyectos FONDECYT 11070091 "Historia poblacional y relaciones biológicas de los habitantes prehistóricos de los oasis atacameños a lo largo de los períodos Formativo Final y Agroalfarero" y National Science Foundation BCS-0721229 "A Bioarchaeological Approach to Identity in the Middle Horizon to Late Intermediate Period Transition in San Pedro de Atacama, Chile". Agradecemos también la asistencia de Blair Daverman en la toma de las muestras para su datación.

\section{$*$ Referencias citadas}

ACUTO, F., 2008. Experiencing Inca domination in Northwestern Argentina and the Southern Andes. Handbook of South American Archaeology, H. Silverman y W. Isbell (Eds.), pp. 845-861. Springer, Nueva York.

ADÁN, L., 1999. Aquellos antiguos edificios. Acercamiento arqueológico a la arquitectura prehispánica tardía de Caspana. Estudios Atacameños 18: 13-33.
ALDEN, J., L. MINC y T. LYNCH, 2006. Identifying the sources of Inka period ceramics from Northern Chile: Results of a neutron activation study. Journal of Archaeological Science 33: 575-594.

BERENGUER, J., A. DEZA, A. ROMÁN y A. LLAGOSTERA, 1986. La secuencia de Myriam Tarrago para San Pedro de Atacama: Un test por termoluminiscencia. Revista Chilena de Antropología 5: 17-24. 
BERENGUER, J., A. ROMÁN, A. DEZA y A. LLAGOSTERA, 1988. Testing a cultural sequence for the Atacama Desert. $\mathrm{Cu}$ rrent Anthropology 29 (2): 341-46.

BRAVO, L. y A. LLAGOSTERA, 1986. Solcor 3: Un aporte al conocimiento de la Cultura San Pedro, período 500 a 900 DC. Chungara 16-17:323-332.

COCILOVO, J. y M. ZAVATTIERI, 1994. Biología del grupo prehistórico de Coyo Oriental (San Pedro de Atacama, norte de Chile): II Deformación craneana artificial. Estudios Atacameños 11: 135-143.

COCILOVO, J., M. ZAVATTIERI y M. A. COSTA, 1994. Biología del grupo prehistórico de Coyo Oriental (San Pedro de Atacama, Norte de Chile): I Dimorfismo sexual y variación etaria. Estudios Atacameños 11: 121-134.

COCILOVO, J., H. VARELA y S. QUEVEDO, 1995. La deformación artificial del cráneo en las poblaciones prehistóricas de San Pedro de Atacama, Chile. Chungara 27 (2): 117-124.

COSTA, M. A., 1985. Quitor 6: Sector Tardío. Memoria para optar al título de Arqueólogo. Departamento de Arqueología, Universidad del Norte, Antofagasta.

1988. Reconstitución física y cultural de la población tardía del cementerio de Quitor 6 (San Pedro de Atacama). Estudios Atacameños 9: 99-126.

COSTA, M. A. y A. LLAGOSTERA, 1994. Coyo 3: Momentos finales del período Medio en San Pedro de Atacama. Estudios Atacameños 11: 73-107.

COSTA, M. A., A. LLAGOSTERA, F. ROTTHAMMER y C. SILVA, 1995. Microdiferenciación craneométrica entre las poblaciones agroalfareras de San Pedro de Atacama, Chile. Hombre y Desierto: Una perspectiva cultural 9: 27-31.

COSTA, M. A., W. NEVES, A. M. BARROS y R. BARTOLOMUCCI, 1998. Trauma y estrés en poblaciones prehistóricas de San Pedro de Atacama, norte de Chile. Chungara 30: 65-74.

COSTA, M. A., W. NEVES y M. HUBBE, 2004. Influencia de Tiwanaku en la calidad de vida biológica de la población prehistórica de San Pedro de Atacama. Estudios Atacameños 27: 103-116.

COSTA, M. A., A. LLAGOSTERA y J. COCILOVO, 2008. La deformación craneana en la población prehistórica de Coyo Oriente, San Pedro de Atacama. Estudios Atacameños 36: 29-41.

COSTA, M. A., C. MATHESON, L. IACHETTA, A. LLAGOSTERA y O. APPENZELLER, 2009. Ancient Leishmaniasis in a highland desert of Northern Chile. PLoS ONE 4: e6983. doi:10.1371/journal.pone.0006983.

HENCKEL, C., 1964. Estudio de cráneos de San Pedro de Atacama y observaciones acerca de la deformación craneana. Boletín de la Sociedad de Biología de Concepción 39: 33-48.

JACOBS, B., 1986. Costumbres mortuorias de la Cultura San Pedro, Atacama, Chile: Estudio de las momias del Museo Arqueológico "R.P. Gustavo Le Paige s.j." Tesis de Magíster, Freien Universität, Berlín.

KNUDSON, K., 2004. Tiwanaku residential mobility in the South Central Andes: Identifying archaeological human migration through Strontium Isotope Analysis. Tesis doctoral, University of Wisconsin, Madison.

2007. La influencia de Tiwanaku en San Pedro de Atacama: Una investigación usando los isótopos del estroncio. Estudios Atacameños 33: 7-24.

2008. Tiwanaku influence in the South Central Andes: Strontium Isotope Analysis and Middle Horizon migration. Latin American Antiquity 19 (1): 3-23.

2009. Oxygen Isotope Analysis in a land of environmental extremes: The complexities of isotopic work in the Andes. International Journal of Osteoarchaeology 19 (2): 171-191.

KNUDSON, K. y D. BLOM, 2009. The complex relationship between Tiwanaku mortuary identity and geographic origin in the South Central Andes. En Bioarchaeology and identity in the Americas, K. Knudson and C. Stojanowski (Eds.), pp. 194-211. University Press of Florida, Tallahassee.

KNUDSON, K. y T. D. PRICE, 2007. Utility of multiple chemical techniques in archaeological residential mobility studies: Case studies from Tiwanaku and Chiribaya affiliated sites in the Andes. American Journal of Physical Anthropology 132 (1): 25-39.

KNUDSON, K. y C. TORRES-ROUFF, 2009. Investigating cultural heterogeneity and multiethnicity in San Pedro de Atacama, Northern Chile, through biogeochemistry and bioarchaeology. American Journal of Physical Anthropology 138 (4): 473-485.

LARRAÍN, C., G. LE PAIGE y R. LARRAÍN DEL CAMPO, 1961. Protocolos craneométricos. Contexto y estudio anatómico de 27 cráneos de la Colección del Museo Arqueológico de San Pedro de Atacama. Anales de la Universidad del Norte 1: 49-110.

LE PAIGE, G., 1964. El precerámico en la cordillera atacameña y los cementerios del período Agroalfarero de San Pedro de Atacama. Anales de la Universidad del Norte 3. 
1966. Cráneos atacameños: Evolución, ritos. Anales de la Universidad del Norte 5: 5-82.

1968-69. Ricardo Latcham y el cementerio indígena de Tchecar. Boletín del Museo de Historia Natural 30: 89-94.

1972-73. Tres cementerios indígenas en San Pedro de Atacama y Toconao. Actas del VI Congreso de Arqueología Chilena (1971). Boletín de Prehistoria, número especial: 163-187.

1973. El valor arqueológico del Museo San Pedro de Atacama. Estudios Atacameños 1: 9-19.

1974. El yacimiento de Tchaputchayna. Estudios Atacameños 2: 59-74.

1977. Recientes descubrimientos arqueológicos en la zona de San Pedro de Atacama. Estudios Atacameños 5: 109-124.

1978. Vestigios arqueológicos incaicos en las cumbres de la zona atacameña. Estudios Atacameños 6: 36-52.

LE PAIGE, G. y C. LARRAÍN, 1961. Estudio craneométrico de la Colección del Museo de San Pedro de Atacama. Anales de la Universidad del Norte 1: 1-48.

LESSA, A., 1995. Estudo de lesões traumáticas agudas como indicadores de tensão social na população do cemitério Solcor 3, San Pedro de Atacama, Chile. MSc Thesis, Escola Nacional de Saúde Pública, Río de Janeiro.

2005. Paleoepidemiologia dos traumas agudos em grupos Atacamenhos: A violência sob uma perspectiva diacrônica. Tesis doctoral, Escola Nacional de Saúde Pública, Río de Janeiro.

LESSA, A. y S. MENDONÇA DE SOUZA, 2003-2004. Paleoepidemiologia dos traumatismos cotidianos em Solcor 3, San Pedro de Atacama, Chile: Riscos diferenciados no período Tiwanaku? Antropología Portuguesa 20-21: 183-207.

2004. Violence in the Atacama Desert during the Tiwanaku period: Social tension? International Journal of Osteoarchaeology 14 (5): $374-388$.

2006. Broken noses for the gods: Ritual battles in the Atacama Desert during the Tiwanaku period. Memorias del Instituto Oswaldo Cruz 101 (2): 133-138.

2007. Gestación de un nuevo panorama social en el oasis atacameño: Conflictos durante la transición para el período de las autonomías regionales. Chungara 39 (2): 209-220.

2009. Invasores a vista: Uma abordagem biocultural sobre a violência durante a Era dos Pukaras no oásis Atacamenho. Revista de Arqueología 22 (2): 105-119.

LLAGOSTERA, A. y M. A. COSTA, 1999. Patrones de asentamiento en la época agroalfarera de San Pedro de Atacama (norte de Chile). Estudios Atacameños 17: 175-206.

LYNCH, T., 1993. The identification of Inca posts and roads from Catarpe to Rio Frio, Chile. En Provincial Inca:Archaeological and ethnohistorical assessment of the impact of the Inca State, M. Malpass (Ed.), pp. 117-142. Iowa University Press, Ciudad de Iowa.

MARSTELLER, S., C. TORRES-ROUFF y K. KNUDSON, 2011. Pre-Columbian Andean sickness ideology and the social experience of Leishmaniasis: A contextualized analysis of bioarchaeological and paleopathological data from San Pedro de Atacama, Chile. International Journal of Paleopathology 1: 24-34.

McCORMAC, F., A. HOGG, P. BLACKWELL, C. BUCK, T. HIGHAM y P. REIMER, 2004. SHCALo4 Southern Hemisphere calibration, o-11.o Cal KYR BP. Radiocarbon 46 (3): 10871092.

MUNIZAGA, J., 1969. Deformación craneana intencional en San Pedro de Atacama. Actas del Congreso Nacional de Arqueología Chilena, pp. 129-134. Museo Arqueológico de La Serena, La Serena.

NEVES, W. y M. A. COSTA, 1998. Adult stature and standard of living in prehistoric San Pedro de Atacama, Northern Chile. $\mathrm{Cu}$ rrent Anthropology 39 (2): 278-281.

NEVES, W., A. M. BARROS y M. A. COSTA, 1999. Incidence and distribution of postcranial fractures in the prehistoric population of San Pedro de Atacama, Northern Chile. American Journal of Physical Anthropology 109 (2): 253-258.

NEVES, W., M. A. COSTA, R. A. SALM y R. CUNHA, 2006. Osteology of social organization in San Pedro de Atacama: Trauma and social tension. Antropología Física Latinoamericana 4: 59-74.

NIELSEN, A., 2002. Asentamientos, conflicto y cambio social en el altiplano de Lípez (Potosí). Revista Española de Antropología Americana 32: 179-205.

NÚÑEZ, L., 1993. Gustavo Le Paige s.j.: Cronología de una misión. Ediciones Universitarias, Antofagasta.

NÚÑEZ, L., C. SANTORO, V. STANDEN y F. TÉLLEZ, 1998. Reflections surrounding the identification of the Virus HTLV-1 in DNA samples from Prehispanic populations from Northern Chile. Estudios Atacameños 15: 99-105. 
ROTTHAMMER, F. y C. SILVA, 1990. Craniometrical variation among South American prehistoric populations: Climatic, altitudinal, chronological, and geographic contributions. American Journal of Physical Anthropology 82 (1): 9-17.

ROTTHAMMER, F., C. SILVA, J. COCILOVO y S. QUEVEDO, 1986. Una hipótesis provisional sobre el poblamiento de Chile, basado en el análisis multivariado de medidas craneométricas. Chungara 16-17: 115-118.

SAUL, F., 1972. The human skeletal remains of Altar de Sacrificios: An osteobiographic analysis. Peabody Museum of Archaeology and Ethnology, Harvard University, Cambridge.

SCHIAPPACASSE, V., V. CASTRO y H. NIEMEYER, 1989. Los Desarrollos Regionales en el Norte Grande. En Culturas de Chile. Prehistoria. Desde sus orígenes hasta los albores de la Conquista, J. Hidalgo, V. Schiappacasse, H. Niemeyer, C. Aldunate e I. Solimano (Eds.), pp. 181-220. Editorial Andrés Bello, Santiago.

STUIVER, M. y P. REIMER, 1993. Extended ${ }^{14} \mathrm{C}$ database and revised CALIB radiocarbon calibration program. Radiocarbon 35 (1): 215-230.

TARRAGÓ, M., 1968. Secuencias culturales de la etapa Agroalfarera de San Pedro de Atacama (Chile). Actas y memorias del XXXVII Congreso Internacional de Americanistas vol. 2: 119-145. Buenos Aires.

TORRES-ROUFF, C., 2002. Cranial vault modification and ethnicity in Middle Horizon San Pedro de Atacama, Chile. Current Anthropology 43 (1): 163-171.

2003. Oral implications of labret use: A case from pre-Columbian Chile. International Journal of Osteoarchaeology 13 (4): 247-251.

2007. La deformación craneana en San Pedro de Atacama. Estudios Atacameños 33: 25-38.

2008. The influence of Tiwanaku on life in the Chilean Atacama: Mortuary and bodily perspectives. American Anthropologist $110(3): 325-337$.

2009. The bodily expression of ethnic identity: Head shaping in the Chilean Atacama. En Bioarchaeology and identity in the Americas, K. Knudson y C. Stojanowski (Eds.), pp. 212-227. University of Florida Press, Gainesville.

TORRES-ROUFF, C. y M. A. COSTA, 2006. Interpersonal violence in prehistoric San Pedro de Atacama, Chile: Behavioral implications of environmental stress. American Journal of Physical Anthropology 130 (1): 60-70.
TORRES-ROUFF, C. y K. KNUDSON, 2007. Examining the life history of an individual from Solcor 3, San Pedro de Atacama: Combining bioarchaeology and archaeological chemistry. Chungara 39 (2): 235-257.

TORRES-ROUFF, C. y L. YABLONSKY, 2005. Cranial vault modification as a cultural artifact: A comparison of the Eurasian steppes and the Andes. Homo: Journal of Comparative Human Bio$\log 56(1): 1-16$

TORRES-ROUFF, C., M. A. COSTA y A. LLAGOSTERA, 2005. Violence in times of change: The Late Intermediate Period in San Pedro de Atacama. Chungara 37 (1): 75-83.

URIBE, M., 2002. Sobre alfarería, cementerios, fases y procesos durante la prehistoria tardía del desierto de Atacama (800-1600 DC). Estudios Atacameños 22: 7-31.

VARELA, H., 1997. La población prehistórica de San Pedro de Atacama: Composición, estructura y relaciones biológicas. Tesis doctoral. Facultad de Ciencias Exactas, Físico-Químicas y Naturales, Universidad Nacional de Río Cuarto, Río Cuarto.

VARELA, H. y J. COCILOVO, 1996. Los componentes de la variación intramuestra en la población prehistórica de San Pedro de Atacama, Chile. Revista Argentina de Antropología Biológica 1 (1): 219-231.

2000. Structure of the prehistoric population of San Pedro de Atacama. Current Anthropology 41 (1): 125-132.

2009. Microevolución en San Pedro de Atacama (norte de Chile): El cementerio de Quitor. Latin American Antiquity 20 (2): 333-342.

VARELA, H., J. COCILOVO y M. A. COSTA, 1990. El dimorfismo sexual en la población prehistórica de San Pedro de Atacama, Chile. Chungara 24-25: 159-166.

VARELA, H., J. COCILOVO y S. VALDANO, 1993. Evaluación de la influencia del efecto sexo, edad y deformación artificial en la estimación de distancias biológicas por medio de la $\mathrm{D}^{2}$ de Mahalanobis. Boletín de la Sociedad Española de Antropología Biológica 14: $135-148$.

VARELA, H., J. COCILOVO y S. QUEVEDO, 1995. La edad como factor de variación intramuestral en la población prehistórica de San Pedro de Atacama, Chile. Chungara 27: 125-134.

VARELA, H., T. O'BRIEN y J. COCILOVO, 2008. The genetic divergence of prehistoric populations of the South-Central Andes as established by means of craniometric traits. American Journal of Physical Anthropology 137 (3): 274-282. 
WEINSTEIN, K., 2001. Comparative skeletal morphology of modern humans and macaques from high and low altitudes. Tesis doctoral, University of Florida, Miami.

2005. Body proportions in ancient Andeans from high and low altitudes.American Journal of Physical Anthropology 128 (3):569-585.
2007. Thoracic skeletal morphology and high-altitude hypoxia in Andean prehistory. American Journal of Physical Anthropology 134 (1): 36-49. 